\title{
Synthesis and Characterization of Photoresponsive Macromolecule for Biomedical Application
}

\author{
Juan Pang, Ziyu Gao, Long Zhang, Huiming Wang and Xiaohong Hu* \\ School of Material Engineering, Jinling Institute of Technology, Nanjing, China
}

Azobenzene, a photo switcher, has attracted increasing interest due to its structural response to photo stimulus in the field of information science and chemical sensing in the recent decades. However, limited water solubility and cytotoxicity restrained their applications in the biomedical field. In research, HA-AZO has been designed as a water soluble photo switcher in biomedical application. Synthesized HA-AZO had good water-solubility and a stable $\pi-\pi^{*}$ transition absorbance peak trans-isomer. With exposure to UV, transformation from trans-isomer to cis-isomer of HA-AZO could be realized according to UV spectra. Reversely, trans-isomer could be gradually recovered from cis-isomer in the dark. Simultaneously, quick response and slow recovery could be detected in the process of structural change. Moreover, repeated illumination was further used to detect the antifatigue property of HA-AZO, which showed no sign of

OPEN ACCESS

Edited by: Baolin Guo,

Xi'an Jiaotong University, China

Reviewed by: Peng Yang, Shaanxi Normal University, China Yaobin Wu,

Southern Medical University, China

${ }^{*}$ Correspondence: Xiaohong $\mathrm{Hu}$ huxiaohong07@163.com

Specialty section:

This article was submitted to Polymer Chemistry, a section of the journal Frontiers in Chemistry

Received: 01 May 2018 Accepted: 25 May 2018 Published: 02 July 2018

Citation:

Pang J, Gao Z, Zhang L, Wang $H$ and HuX (2018) Synthesis and Characterization of Photoresponsive Macromolecule for Biomedical Application. Front. Chem. 6:217. doi: 10.3389/fchem.2018.00217 fatigue during 20 circles. The influence of $\mathrm{pH}$ value on UV spectrum for HA-AZO was investigated in the work. Importantly, in acid solution, HA-AZO no longer showed any photoresponsive property. Additionally, the status of HA-AZO under the effect of UV light was investigated by DLS results and TEM image. Finally, in vitro cytotoxicity evaluations were performed to show the effects of photoresponsive macromolecule on cells.

Keywords: azobenzene, photo switcher, photoresponsive macromolecule, biomedical application, hyaluronic acid

\section{INTRODUCTION}

Photoresponsive molecules, which cause reversible changes, i.e., their chemical and physical properties changes due to structural change with a light stimulus, have attracted increasing interest in the field of information science and chemical sensing in the recent decades (Sun et al., 2012; Li et al., 2014; Yuan et al., 2014; Kim et al., 2015). Among photo molecular switches, azobenzenes have been intensively investigated due to the precise spatiotemporal control (Li et al., 2014; Kim et al., 2015). Upon exposure to UV light, azobenzenes can isomerize from the trans-form to the cis-form (Henzl et al., 2006; Kumar and Suresh, 2009; Schmidt et al., 2010). However, the cis-form is unstable and liable to reversely isomerize to trans-form under the action of visible light or slight heat (Henzl et al., 2006; Kumar and Suresh, 2009; Schmidt et al., 2010). Given the fact that cis- and trans-isomers of azobenzenes have a different spatial arrangement of the aromatic moieties, physical and chemical properties of azobenzenes varied a lot, some examples being $\pi-\pi$ stack interaction among molecules, dipole moments, and surface wettability (Li et al., 2014; Yuan et al., 2014; Lin et al., 2016, 2017). Consequently, azobenzenes became particularly popular as photoresponsive chromophores in a number of research fields including biomedical fields (Deka et al., 2015; Liu et al., 2015; Bian et al., 2016a,b). Recently, on account of photo-controlled reversible supermolecular interaction between 
azobenzenes and cyclodextrins, hydrogels as well as films with controllable pores or passages crosslinked by the supermolecular interaction have been designed and synthesized to respond to photo-stimuli from external environment (Chen et al., 2009; Chiang and Chu, 2015; Wang et al., 2015). In addition to these reports, visible light driven azobenzene-based photo-switching molecules have been theoretically designed by different substitute groups (Pang et al., 2014, 2016; Ye et al., 2015).

On account of aqueous physiological environments, chemicals in the application of biomedicine-related fields should have aqueous dissolvability (Gao et al., 2016; Wang et al., 2016; Gu et al., 2017; Ha et al., 2018). From this point, the application of azobenzene in the biomedicine-related field is restricted not only due to its low aqueous dissolvablity but also due to cytotoxicity in water. In order to solve this problem, a watersoluble photo switch was designed. From previous research, some natural polysaccharides such as hyaluronic acid (HA), chitosan, chondroitin sulfate (CS) and the like is proven to be water soluble and have good biocompatibility especially for HA, which plays an important role in the organization and stabilization of ECM, cell proliferation, and differentiation (Hu et al., 2011; Hu and Gong, 2016). Moreover, HA can lubricate joints, adjust permeability of vessel walls, improve transport of protein and ions, and accelerate wound healing (Yue et al., 2017; Andreasen et al., 2018). Hence, $\mathrm{HA}$ is assumed to be an ideal macromolecule or material in biomedical application. In view of these facts, a photoresponsive macromolecule was designed using $\mathrm{HA}$ as a backbone and AZO as a side chain in this work.

In consideration of reactivity and carboxyl groups on HA molecules, p-aminoazobenzene was grafted onto the main chain of HA to obtain a photoresponsive macromolecule. In order to clarify the properties of the photosensitive macromolecules, molecular responsive properties as a function of irradiation time, $\mathrm{pH}$ value, and recovered time as well as fatigue resistance of the responsive property was characterized in the research. As a macromolecule, its molecular aggregation in water is also important to evaluate the interaction between molecules and provide potential material for biomedical application. Therefore, the macromolecule with different responsive status in water was tracked by dynamic light scattering (DLS) and molecular aggregation in water was also confirmed by transmission electron microscope (TEM) images. Finally, cell viability and apoptosis profile was used to evaluate the cytotoxicity of photoresponsive macromolecule.

Although azobenzenes have been intensively investigated, work still needs to be done to obtain a biocompatible photoresponsive macromolecule for biomedical application, characterize their structure and assess their properties including cytotoxicity properties.

\section{EXPERIMENTAL SECTION}

\section{Materials}

1-Ethyl-3-(3-dimethylaminopropyl) carbodiimide hydrochloride (EDC), N-hydroxysuccinimide (NHS), 2-morpholinoethane sulfonic acid (MES), and p-aminoazobenzene (AZO) was purchased from Aladdin. Hyaluronate acid $\left(\mathrm{HA}, \mathrm{M}_{\mathrm{W}}=1,000\right.$ $\mathrm{kDa}$ ) was obtained from Shandong Furuida Co., China. Trypsin,
Dulbecco's modified Eagle's medium (DMEM), fluorescein diacetate (FDA), and 3-(4, 5-dimethyl) thiazol-2,5-dimethyl tetrazolium bromide (MTT) were obtained from Sigma. Fetal bovine serum (FBS) was purchased from Sijiqing biotech. Co., China. All other reagents and solvents were of analytical grade and used as received.

\section{Synthesis of HA-AZO}

HA-AZO was synthesized by amidation between $\mathrm{HA}$ and aminoazobenzene (AZO). Briefly, $500 \mathrm{mg}$ of EDC and $200 \mathrm{mg}$ of NHS were successively added to $10 \mathrm{~mL}$ of $2 \% \mathrm{HA}$ solution with magnetic stirring. Meanwhile, $10 \mathrm{mg}$ of AZO was dissolved in $10 \mathrm{~mL}$ DMF. After $30 \mathrm{~min}, 100 \mathrm{mg}$ of MES was further added to HA solution. HA solution and AZO solution were subsequently mixed together and the reaction was maintained for $5 \mathrm{~h}$ at room temperature. Then, the final solution was dialyzed with a dialysis bag of $10 \mathrm{kDa}$ cut-off molecular weight for 3 days to remove unreacted chemicals and byproduct of small molecular weight. Finally, HA-AZO was obtained by freeze drying at $-60^{\circ} \mathrm{C}$ at a pressure of 7-8 $\mathrm{Pa}$.

\section{Characterization of HA-AZO}

$\mathrm{HA}-\mathrm{AZO}$ was characterized by ${ }_{1} \mathrm{H}$ nuclear magnetic resonance $\left({ }_{1} \mathrm{H}\right.$ NMR, Bruker, AV500) using $\mathrm{D}_{2} \mathrm{O}$ as solvent. HA-AZO water solution, AZO water solution, and AZO DMSO solution was characterized by UV spectroscopy (Cary 50). UV lamp (10 W) was used as photosource to induce tran-to-cis transition of AZO domain. In order to track the structural change of molecules, realtime UV spectra as a function of irradiation time and recovery time was recorded. Repeated irradiation and recovery method were applied to demonstrate the fatigue resistance of molecules. Macromolecule status in water solution was characterized by dynamic light scattering (DLS, nano ZS) and transmission electron microscope (TEM, Philips, Tecnai 12).

\section{Cytotoxicity Evaluation of HA-AZO}

HUVEC cells were incubated in a humidified atmosphere of $95 \%$ air and $5 \% \mathrm{CO}_{2}$ at $37^{\circ} \mathrm{C}$. The used cells were detached using $0.25 \%$ trypsin in PBS for the experiment. Simultaneously, HA-AZO and AZO were dissolved in DMEM with certain concentration separately with the same molar ratio. Then, $100 \mu \mathrm{L}$ of above-mentioned solution was added into each well of 96-well culture plate, and into which the $100 \mu \mathrm{L}$ cell suspension containing 5,000 cells was subsequently added. Cells were characterized as a function of cultural time. MTT assay was used to characterize cytoviability. Briefly, $20 \mu \mathrm{L}$ MTT was supplemented into wells of the culture plate, which were then put back to continually culture cells for another $4 \mathrm{~h}$. Two hundred microliters DMSO was added to dissolve the formed formazan pigment. The absorbance of above solution at $490 \mathrm{~nm}$ was recorded by a microplate reader (Infinite M200 PRO).

Besides MTT assay, apoptosis profile of cells was characterized by PI/FITC double staining (Annexin V). Briefly, $1 \mathrm{~mL}$ of abovementioned HA-AZO solution or AZO solution was added into each well of 12-well culture plate, into which $1 \mathrm{~mL}$ cell suspension containing 200,000 cells was subsequently added. Cells of each well were detached using $0.25 \%$ trypsin without EDTA after culturing for $24 \mathrm{~h}$ separately. Detached cells were washed with 
$1 \times$ binding buffer, resuspended in $500 \mu \mathrm{L} 1 \times$ binding buffer, and subsequently ordinal stained by PI solution and FITC solution for $15 \mathrm{~min}$. The stained cells detected by flow cytometry (FCM, BD, C6) within $1 \mathrm{~h}$ in dark.

\section{Statistical Analysis}

Data were analyzed using the $t$-test for differences. Results were reported as means \pm standard deviation. The significant level was set at $p<0.05$.

\section{RESULT AND DISCUSSION}

\section{Synthesis of HA-AZO}

Synthesized HA-AZO was characterized by ${ }_{1} \mathrm{H}$ NMR as shown in Figure 1. The details of chemical shift are listed as follows: the chemical shift at $1.9 \mathrm{ppm}$ is attributed to the protons of $\mathrm{CH}_{3}-\mathrm{O}$ at 1 position, the chemical shifts from 3.1 to $4.0 \mathrm{ppm}$ are attributed to the protons of pyranose ring, and the chemical shift at $7.9 \mathrm{ppm}$ is attributed to the protons of benzene ring of $\mathrm{AZO}$ at 2 position. The chemical shift at 2 position confirmed successful grafting of AZO onto HA main chain. Besides qualitative analysis, ${ }_{1} \mathrm{H}-$ NMR provided quantitative information since areas of resonance peaks are proportional to number of protons. According to areas in Figure 1 at 1 and 2 positions, the degree of substituent (DS) could be calculated using the average proton intensity ratio of the protons of benzene ring/ $\mathrm{CH}_{3}-\mathrm{O}$, which is $2.2 \%$ (per two pyranose ring).

\section{Characterization of HA-AZO}

It was found that HA-AZO had good water-solubility. Hence, HA-AZO water solution was used for the following characterization. Firstly, UV spectra of HA-AZO water solution as a function of irradiation time and recovery time were shown in Figures 2A,B. In one aspect, the peak at $360 \mathrm{~nm}$ belonging to $\pi-\pi^{*}$ transition for trans-isomer decreased significantly and the peak at $420 \mathrm{~nm}$ belonging to $n-\pi^{*}$ transition for cis-isomer increased a little with irradiation time until $60 \mathrm{~s}$, which was an obvious sign to indicate that the trans-form had been transferred to the cis-form (Figure 2A). In another aspect, without irradiation, the peaks at 360 and $420 \mathrm{~nm}$ were gradually recovered to their original state within $10 \mathrm{~min}$, which indicated that the recovery of trans-form for AZO domain of HA-AZO was realized (Figure 2B). Structural change upon photo guaranteed the molecular responsive property for HAAZO. Moreover, rapid transfer from trans to cis upon photo exhibited quick responsive time for the macromolecule, and simultaneously gradual recovering process indicated enough controllable time. Effective structural change, rapid responsive time, and enough operation time are all desirable properties for a macromolecule photo switch. As a contrast, UV spectra of AZO water solution showed no change after irradiation without any change of isomer (Figure s1), although AZO DMF solution showed some photo-responsive properties (Figure s2). Secondly, fatigue resistance of molecular switch was essential for its actual application, which was evaluated by repeated irradiation and recovery and characterized by UV spectrum as shown in Figures 2C,D. It was found that the minimum absorbance at $346 \mathrm{~nm}$ for HA water solution after irradiation was stabilized at $0.7-0.8$ regardless of circle time, and simultaneously maximum absorbance at $360 \mathrm{~nm}$ either initial or after recovery was stabilized at 1.5-1.7 regardless of circle time (Figure 2C). The results showed that cis-form could be stable and exist after irradiation, whereas the trans-form could be recovered after removal of irradiation. Furthermore, the response time

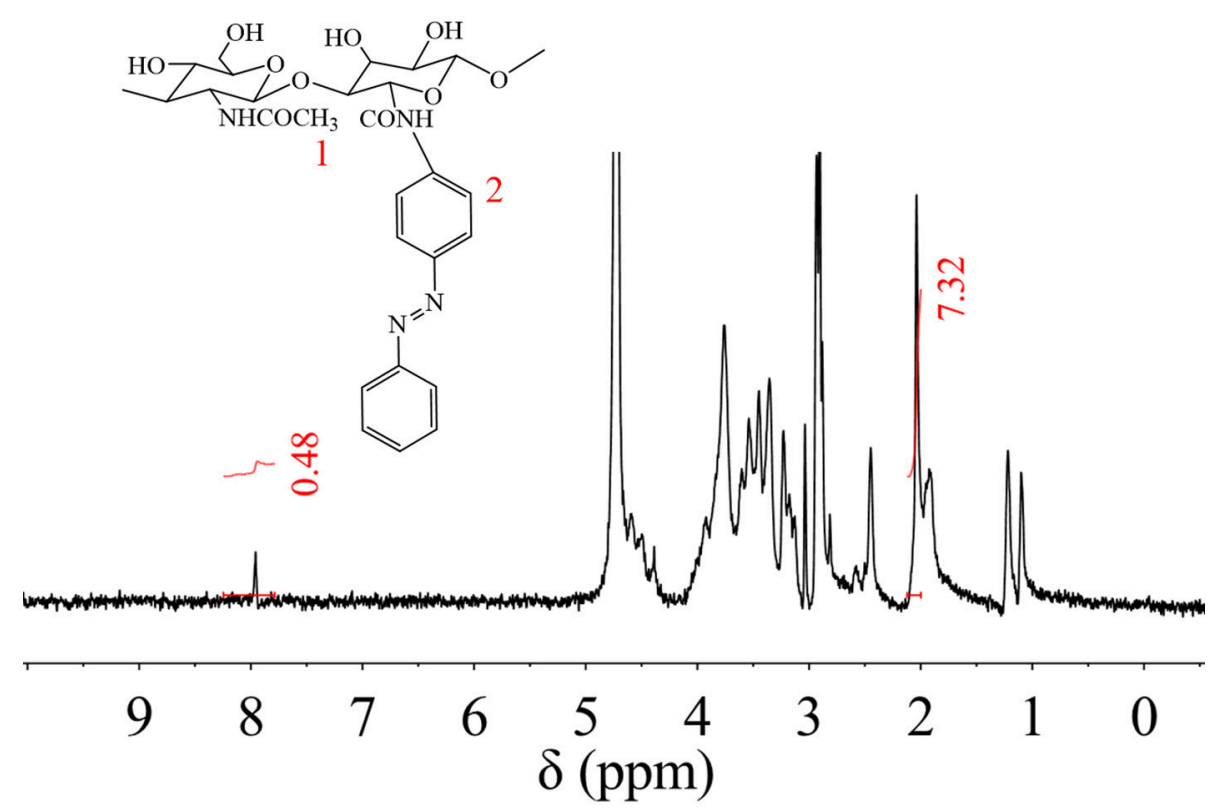

FIGURE $1 \mid{ }_{1} \mathrm{H}$ NMR spectrum of HA-AZO. 

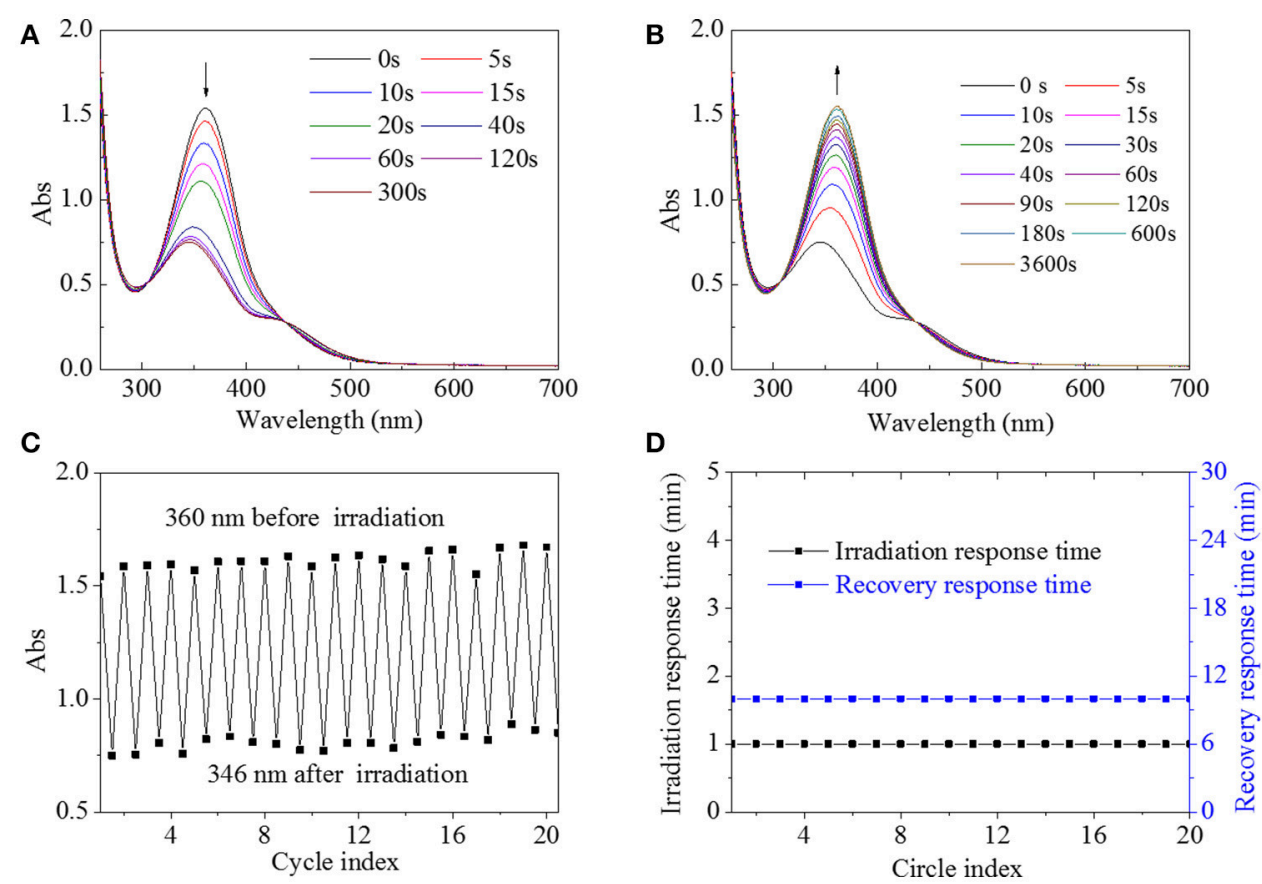

FIGURE 2 | UV spectra of HA-AZO water solution as a function of irradiation time (A) and recovery time (B). (C) Absorbance at 360/346 nm of HA-AZO water solution as a function of circle index. (D) Irradiation response time and recovery response time in dark as a function of circle index.
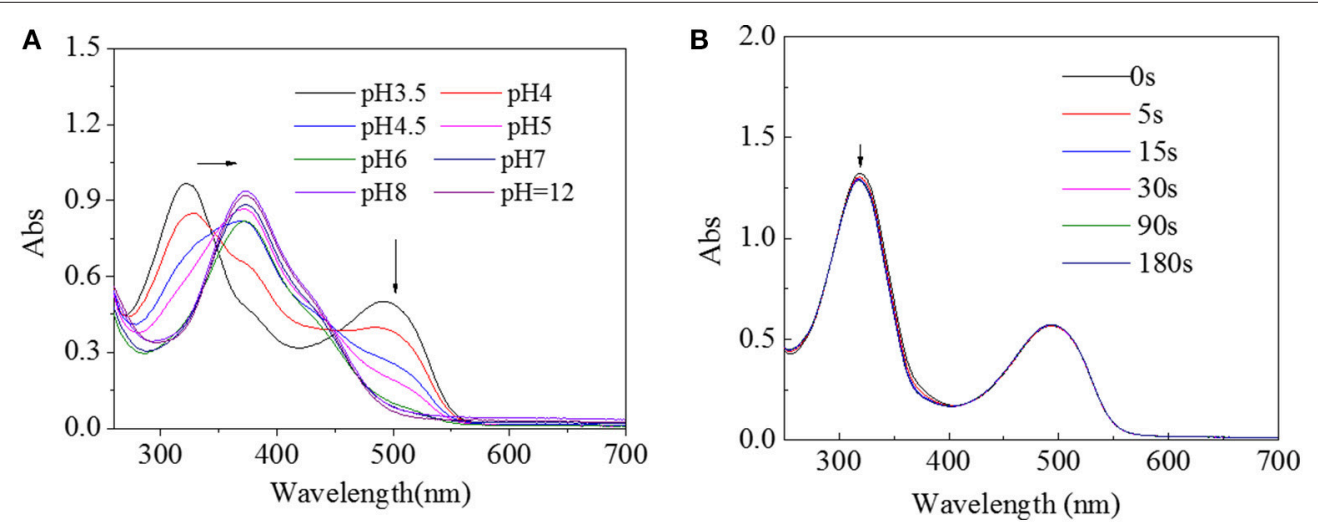

FIGURE 3 | (A) UV spectra of HA-AZO water solution as a function of $\mathrm{pH}$ value. (B) UV spectra of HA-AZO water solution as a function of irradiation time in $\mathrm{pH}=3.5$ medium.

with irradiation was stable at $1 \mathrm{~min}$, and the recovery time was stable at $10 \mathrm{~min}$ regardless of circle time (Figure 2D). From these results, it is inferred that HA-AZO has no sign of fatigue or phenomenon of photobleaching. As a contrast, even in DMF, the photoresponsive property weakened as a function of circle time (Figure s3A), and furthermore, the response time and recovery time shortened with increase of circle time (Figure s3B), which was unfavorable for its application as a photo switch. By contrast, HA-AZO had obvious superiority to AZO molecule as a photo switch, especially in water solution.
Since $\mathrm{pH}$ value is also an important factor in physiological environment, UV spectra of HA-AZO solution with different $\mathrm{pH}$ value was tracked as shown in Figure 3A. With decreasing $\mathrm{pH}$ value, the peak at $360 \mathrm{~nm}$ shifted to $320 \mathrm{~nm}$ and a new peak at $500 \mathrm{~nm}$ gradually increased when $\mathrm{pH}$ value was lower than 6 . The blue shift from 360 to $320 \mathrm{~nm}$ was estimated to protonation effect of benzene ring for $\pi-\pi^{*}$ transition and emerging peak at $500 \mathrm{~nm}$ was estimated to $\mathrm{n}-\pi^{*}$ transition in effects of protonation. In addition, in order to inspect the influence of irradiation on structure of HA-AZO, UV spectra of acid HA-AZO solution as a function of irradiation 

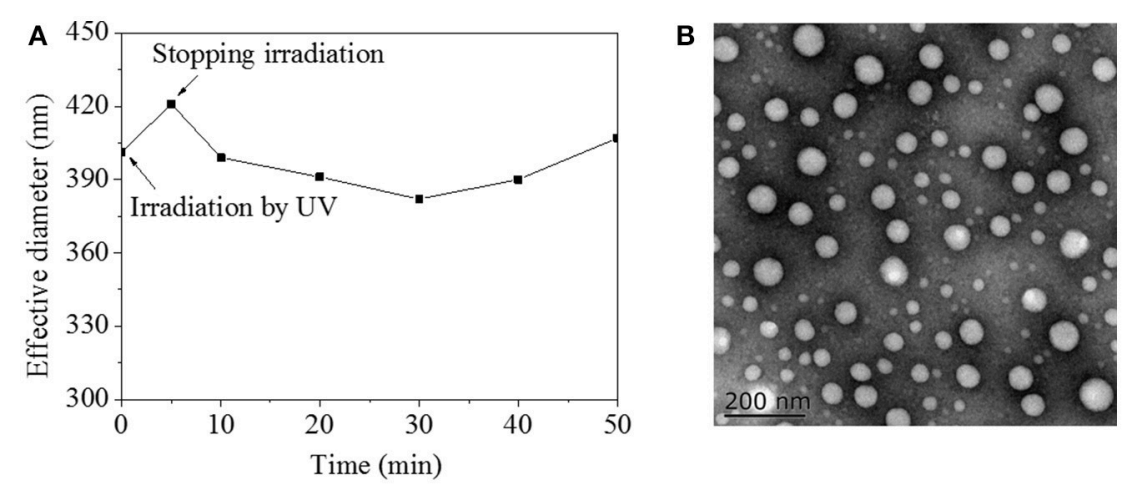

FIGURE 4 | (A) Effective diameter of HA-AZO water solution a function of time by influence of UV irradiation; (B) TEM images of HA-AZO dropped by HA-AZO water solution and dried naturally.

time was detected as shown in Figure 3B. It was found that the macromolecule has no responsive property in acid environment since UV irradiation had no influence on its UV spectra, which indicated that HA-AZO exhibited $\mathrm{pH}-$ dependent photoresponsive property. Theoretically, no visible trans-to-cis transition in acid environment might be the case, for two reasons. One is unstability of cis-form structure with very short existing time beyond characterization. The other one is protonation of AZO induces cis-form structure (from $\mathrm{n}-\pi^{*}$ transition) that coexists with trans-form structure in natural state, which indicates that trans-to-cis transition is induced in acid environment. However, no defined deduction has been verified by sufficient proof. Furthermore, since AZO molecule in water exhibited no trans-to-cis transition due to aggregation of molecules according to Figure S1, AZO molecule in water could not have $\mathrm{pH}$-dependent photo responsive property.

On account of $\pi-\pi$ stacking interaction between AZO domains, HA-AZO macromolecule might be in aggregation state, which was characterized by DLS and TEM as shown in Figure 4. Before irradiation, the effective diameter of HAAZO solution was $405 \mathrm{~nm}$ (Figure 4A). The effective diameter increased after irradiation and then decreased gradually along with time until $30 \mathrm{~min}$ (Figure 4A). According to previous research, $\pi-\pi$ stacking interaction between AZO molecules could be disaggregated when the trans-form transferred to cis-form, which made the interaction within aggregations weakened. The weaker interaction could lead aggregations to swell and dismiss at the end. However, since chain movement of macromolecule need time, recovery process of cis to trans came to procedure before aggregations dismissed completely. In recovery procedure, dismissed aggregations gradually reassembled again along with time. In further step, HA-AZO aggregations were observed by TEM as shown in Figure 4B. Spherical aggregations with diameter of 20$100 \mathrm{~nm}$ were homogeneously distributed in the TEM image. Because HA-AZO aggregations in water were in swollen state, diameter detected by DLS was much larger than that detected by TEM.

\section{Cytotoxicity of HA-AZO}

Since biocompatibility of the macromolecule was an essential factor for its biomedical application, preliminarily cytotoxicity assessment was performed by in vitro culture of HUVEC cells. In this method, cells were cocultured with HA-AZO on TCPs, and simultaneously, cytoviability and cell apoptosis profiles were used to evaluate the biocompatibility of HAAZO. As shown in Figure 5, the viability of cells decreased with HA-AZO concentration regardless of culture time. On the first day, OD of cells with HA-AZO showed no obvious decline, estimated $80 \%$ of that on TCPs even when HA-AZO concentration was as high as $5 \mathrm{mg} / \mathrm{mL}$, which indicated that HA-AZO had no obvious acute cytotoxicity. However, cells with HA-AZO showed much lower OD than cells without HA-AZO after cells were cultured for some time. Moreover, OD of cells increased with cultured time, which indicated that cells proliferated along with time. However, it was inferred from OD statistic data that cells with HA-AZO showed slower proliferation rate than that of control (without HA-AZO), and the proliferation rate decreased along with HA-AZO concentration. These results indicated that proliferation of cells might be inhibited by HA-AZO. As a contrast, equivalent AZO exhibited great acute cytotoxicity and complete proliferation inhibition (Figure s4).

In the cell apoptosis profile, live cells on TCPs without HAAZO were approaching $78 \%$ and the dead cells were estimated to be $18 \%$ after cells were cultured for 3 days (Figure 6A). Further live cells on TCPs decreased with the increase of HA-AZO concentration (Figure 6B-D). Even when HA-AZO concentration was $5 \mathrm{mg} / \mathrm{mL}$, live cells had been estimated $60 \%$. The results were consistent with their viability as shown in Figure 5. As a contrast, equivalent AZO exhibited great cytotoxicity with less live cells and more dead cells in Figure s5. 

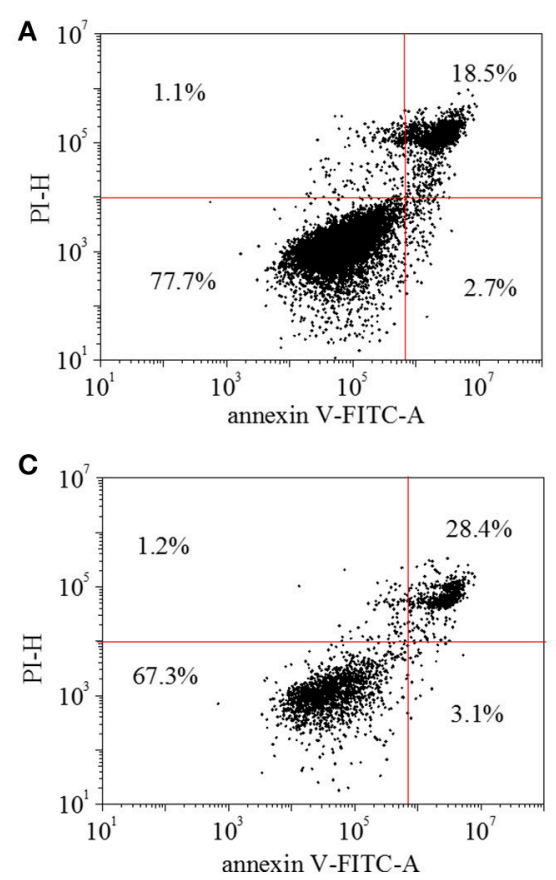
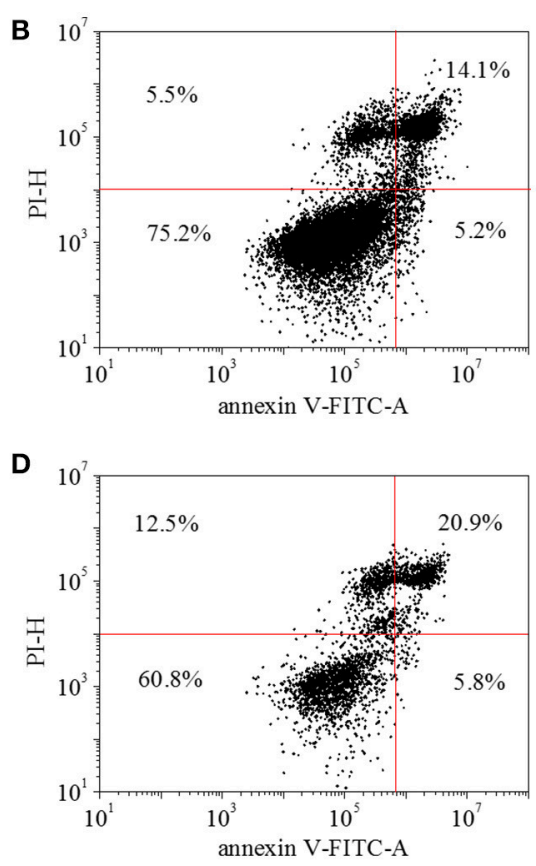

FIGURE 6 | HUVEC cell apoptosis profile of with (A) 0 mg/mL HA-AZO, (B) 1 mg/mL HA-AZO, (C) 5 mg/mL HA-AZO, (D) 10 mg/mL HA-AZO. Cells was stained by FITC/PI.

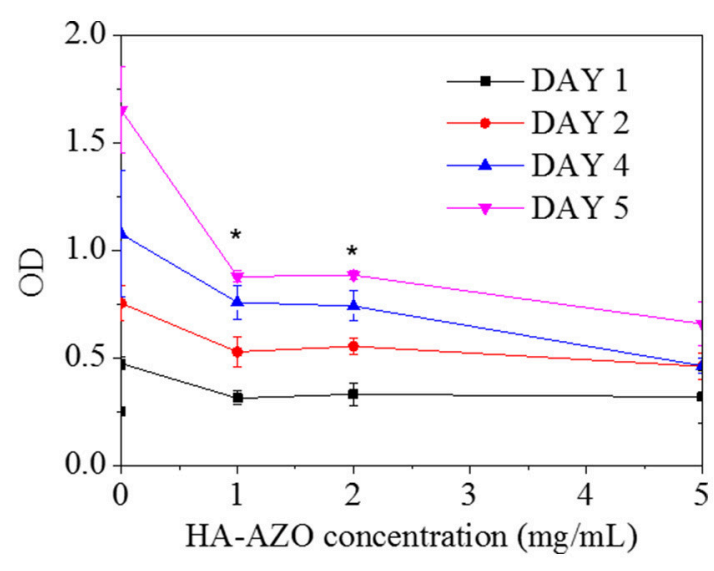

FIGURE 5 | Optical density of HUVEC cells after cultured different time as a function of HA-AZO concentration. Cells was incubated with MTT.

\section{CONCLUSION}

HA-AZO was successfully synthesized with DS of $2.2 \%$. Synthesized HA-AZO had good water-solubility and a stable $\pi-\pi^{*}$ transition absorbance peak trans-isomer. Upon UV for $5 \mathrm{~min}$, transformation from trans-isomer to cis-isomer of HAAZO could be realized according to UV spectra. Reversely, trans-isomer could be gradually recovered from cis-isomer in $1 \mathrm{~h}$ in dark. This characteristics revealed the reversible photoresponsive property of HA-AZO molecule with quick response and slow recovery. Moreover, antifatigue property of HA-AZO was confirmed by repeated illumination. The blue shift from 360 to $320 \mathrm{~nm}$ and emerging peak at $500 \mathrm{~nm}$ were found in low $\mathrm{pH}$ value solution. In acid solution, HA-AZO no longer showed any photo responsive property. HA-AZO was in small aggregation and its size was influenced by irradiation and varied with recovery time. Finally, in vitro evaluation showed little acute cytotoxicity, but the cell growth could be inhibited by HA-AZO. These properties indicated that HA-AZO was an optimal photo responsive macromolecule for biomedical application. The toxicity of AZO has been verified not only by our results but also by other researchers. Hence, their application in biomedical field is rarely reported. While the AZO grafting product HA-AZO exhibits little acute cytotoxicity from the result of both cell viability and cell apoptosis profile after $1 \mathrm{~d}$, acute cytotoxicity is evaluated with $24 \mathrm{~h}$ before cell division. Theoretically, cell viability should multiply and increase with cultured days, since cells divide in every $24 \mathrm{~h}$. Actually, cell growth viability showed little increase with cultured days. However, the increased rate is much lower than both that of TCPs control and theoretical calculation. These results showed that HA-AZO influenced cell proliferation and cell division, which indicated that the grafting product might have some genetic toxicity.

\section{AUTHOR CONTRIBUTIONS}

JP designed the macromolecule and predicted its property. ZG synthesized the macromolecule. LZ and HW investigated the 
properties of the macromolecule. $\mathrm{XH}$ was responsible for the whole manuscript.

\section{ACKNOWLEDGMENTS}

This study was financially supported by Natural Science Foundation of (21702082), Natural Science Foundation of Jiangsu Province (BK20171113), Qing Lan Project, the

\section{REFERENCES}

Andreasen, C. M., Ding, M., Andersen, T. L., and Overgaard, S. (2018). Effects of substitute coated with hyaluronic acid or poly-lactic acid on implant fixation: Experimental study in ovariectomized and glucocorticoidtreated sheep. J. Tissue Eng. Regener. Med. 12, e1122-e1130. doi: 10.1002/ term. 2447

Bian, Q., Wang, W., Han, G., Chen, Y., Wang, S., and Wang, G. (2016a). Photoswitched cell adhesion on azobenzene-containing self-assembled films. Chemphyschem 17, 2503-2508. doi: 10.1002/cphc.201600362

Bian, Q., Wang, W., Wang, S., and Wang, G. (2016b). Light-triggered specific cancer cell release from cyclodextrin/azobenzene and aptamermodified substrate. ACS Appl. Mater. Interfaces 8, 27360-27367. doi: $10.1021 /$ acsami.6b09734

Chen, X., Hong, L., You, X., Wang, Y., Zou, G., Su, W., et al. (2009). Photocontrolled molecular recognition of $\alpha$-cyclodextrin with azobenzene containing polydiacetylene vesicles. Chem. Commun. 1356-1358. doi: 10.1039/b820894h

Chiang, C. Y., and Chu, C. C. (2015). Synthesis of photoresponsive hybrid alginate hydrogel with photo-controlled release behavior. Carbohydr. Polym. 119, 18-25. doi: 10.1016/j.carbpol.2014.11.043

Deka, S. R., Yadav, S., Mahato, M., and Sharma, A. K. (2015). Azobenzeneaminoglycoside: self-assembled smart amphiphilic nanostructures for drug delivery. Colloids Surf. B Biointerfaces 135, 150-157. doi: 10.1016/j.colsurfb.2015.07.026

Gao, A., Wu, Q., Wang, D., Ha, Y., Chen, Z., and Yang, P. (2016). A superhydrophobic surface templated by protein self-assembly and emerging application toward protein crystallization. Adv. Mater. 28, 579-587. doi: 10.1002/adma.201504769

Gu, J., Su, Y., Liu, P., Li, P., and Yang, P. (2017). An environmentally benign antimicrobial coating based on a protein supramolecular assembly. ACS Appl. Mater. Interfaces 9, 198-210. doi: 10.1021/acsami.6b13552

Ha, Y., Yang, J., Tao, F., Wu, Q., Song, Y. J.,Wang, H., et al. (2018). Phase-transited lysozyme as a universal route to bioactive hydroxyapatite crystalline film. $A d v$. Funct. Mater. 28:1704476. doi: 10.1002/adfm.201704476

Henzl, J., Mehlhorn, M., Gawronski, H., Rieder, K. H., and Morgenstern, K. (2006). Reversible cis-trans isomerization of a single azobenzene molecule. Angew. Chem. 45, 603-606. doi: 10.1002/anie.200502229

$\mathrm{Hu}, \mathrm{X}$., and Gong, X. (2016). A new route to fabricate biocompatible hydrogels with controlled drug delivery behavior. J. Colloid Interface Sci. 470, 62-70. doi: 10.1016/j.jcis.2016.02.037

Hu, X., Li, D., Zhou, F., and Gao, C. (2011). Biological hydrogel synthesized from hyaluronic acid, gelatin and chondroitin sulfate by click chemistry. Acta Biomater. 7,1618-1626. doi: 10.1016/j.actbio.2010.12.005

Kim, D. Y., Lee, S. A., Park, M., Choi, Y. J., Kang, S. W., and Jeong, K. U. (2015). Multi-responsible chameleon molecule with chiral naphthyl and azobenzene moieties. Soft Matter 11, 2924-2933. doi: 10.1039/C5SM00073D

Kumar, B., and Suresh, K. A. (2009). Kinetics of trans-cis isomerization in azobenzene dimers at an air-water interface. Phys. Rev. E Stat. Nonlinear Soft Matter Phys. 80:021601. doi: 10.1103/PhysRevE.80.021601

Li, Z., Wang, P., Liu, B., Wang, Y., Zhang, J., Yan, Y., et al. (2014). Unusual, photoinduced self-assembly of azobenzene-containing amphiphiles. Soft Matter 10, 8758-8764. doi: 10.1039/C4SM01395F

Lin, L. R., Tang, H. H., Wang, Y. G., Wang, X., Fang, X. M., and Ma, L. H. (2017). Functionalized Lanthanide(III) complexes constructed from azobenzene derivative and beta-diketone ligands: luminescent, magnetic,
Scholarship of Jiangshu Government, Six talent peaks project in Jiangsu Province (JY-071).

\section{SUPPLEMENTARY MATERIAL}

The Supplementary Material for this article can be found online at: https://www.frontiersin.org/articles/10.3389/fchem. 2018.00217/full\#supplementary-material

and reversible trans-to-cis photoisomerization properties. Inorg. Chem. 56, 3889-3900. doi: 10.1021/acs.inorgchem.6b02819

Lin, L. R., Wang, X., Wei, G. N., Tang, H. H., Zhang, H., and Ma, L. H. (2016). Azobenzene-derived tris-beta-diketonate lanthanide complexes: reversible trans-to-cis photoisomerization in solution and solid state. Dalton Trans. 45, 14954-14964. doi: 10.1039/C6DT01310D

Liu, G. F., Ji, W., Wang, W. L., and Feng, C. L. (2015). Multiresponsive hydrogel coassembled from phenylalanine and azobenzene derivatives as 3D scaffolds for photoguiding cell adhesion and release. ACS Appl. Mater. Interfaces 7, 301-307. doi: 10.1021/am506202s

Pang, J., Tian, Z. Q., and Mab, J. (2014). Theoretical design of visible light driven azobenzene-basedphoto-switching molecules. Chem. Phys. Lett. 613, 110-114. doi: 10.1016/j.cplett.2014.07.048

Pang, J., Ye, Y. F., Tian, Z. Q., Pang, X. Y., and Wu, C. Y. (2016). Theoretical insight into azobis-(benzo-18-crown-6) ether combined with the alkaline earth metal cations. Comput. Theor. Chem. 1076, 17-22. doi: 10.1016/j.comptc.2015. 04.012

Schmidt, R., Hagen, S., Brete, D., Carley, R., Gahl, C., Dokic, J., et al. (2010). On the electronic and geometrical structure of the trans- and cis-isomer of tetratert-butyl-azobenzene on Au(111). Phys. Chem. Chem. Phys. 12, 4488-4497. doi: 10.1039/b924409c

Sun, Y., Yu, C., Liu, Z., Huang, C., Hao, Q., and Xu, L. (2012). Synthesis, structure, photo-responsive properties of 4-(2-fluorobenzylideneamino)antipyrine: a combined experimental and theoretical study. Spectrochim. Acta A Mol. Biomol. Spectrosc. 97, 1013-1022. doi: 10.1016/j.saa.2012.07.117

Wang, D., Ha, Y., Gu, J., Li, Q., Zhang, L., and Yang, P. (2016). 2D protein supramolecular nanofilm with exceptionally large area and emergent functions. Adv. Mater. 28, 7414-7423. doi: 10.1002/adma.201506476

Wang, D., Wagner, M., Butt, H. J., and Wu, S. (2015). Supramolecular hydrogels constructed by red-light-responsive host-guest interactions for photo-controlled protein release in deep tissue. Soft Matter 11, 7656-7662. doi: 10.1039/C5SM01888A

Ye, Y. F., Pang, J., Zhou, X. J., and Huang, J. W. (2015). Understanding the torsion effects on optical properties of azobenzene derivatives. Comput. Theor. Chem. 1066, 28-33. doi: 10.1016/j.comptc.2015.11.022

Yuan, K., Guo, Y. J., and Zhao, X. (2014). A novel photo-responsive azobenzene-containing nanoring host for fullerene-guest facile encapsulation and release. Phys. Chem. Chem. Phys. 16, 27053-27064. doi: 10.1039/C4CP0 $3687 \mathrm{E}$

Yue, C., Sun, H., Liu, W. J., Guan, B., Deng, X., Zhang, X., et al. (2017). Environmentally benign, rapid, and selective extraction of gold from ores and waste electronic materials. Angew. Chem. 56, 9331-9335. doi: 10.1002/anie.201703412

Conflict of Interest Statement: The authors declare that the research was conducted in the absence of any commercial or financial relationships that could be construed as a potential conflict of interest.

Copyright (c) 2018 Pang, Gao, Zhang, Wang and Hu. This is an open-access article distributed under the terms of the Creative Commons Attribution License (CC BY). The use, distribution or reproduction in other forums is permitted, provided the original author(s) and the copyright owner(s) are credited and that the original publication in this journal is cited, in accordance with accepted academic practice. No use, distribution or reproduction is permitted which does not comply with these terms. 